\title{
A Novel Unsupervised Abnormal Event Identification Mechanism for Analysis of Crowded Scene
}

\author{
Dr. Pushpa D \\ Department of Information Science and Engineering, \\ Maharaja Institute of Technology, Mysuru, India
}

\begin{abstract}
The advancement of visual sensing has introduced better capturing of the discrete information from a complex, crowded scene for assisting in the analysis. However, after reviewing existing system, we find that majority of the work carried out till date is associated with significant problems in modeling event detection as well as reviewing abnormality of the given scene. Therefore, the proposed system introduces a model that is capable of identifying the degree of abnormality for an event captured on the crowded scene using unsupervised training methodology. The proposed system contributes to developing a novel region-wise repository to extract the contextual information about the discrete-event for a given scene. The study outcome shows highly improved the balance between the computational time and overall accuracy as compared to the majority of the standard research work emphasizing on event detection.
\end{abstract}

Keywords-Abnormal event; detection; event detection; object detection; machine learning; video surveillance

\section{INTRODUCTION}

With the evolution of visual sensors, the security and monitoring based application has been consistently witnessing revolution [1]. Such advanced forms of image capturing devices offers enhanced capability to obtain various scenic information but is also shrouded by various loopholes. The first problem lies in identifying the moving objects on the given scene. At present, there are various research work that has contributed towards object detection, tracking, and counting [2] but none of these actually existing in commercial application of global market at this time. We find that there is a bigger gap between the research papers and application existing in realtime scenario [3]. The existing research work claims of using sophisticated theory and technologies to perform extraordination tracking and identification of an object but in realtime environment there are various applications which have never seen such implementations ever. Some of the impediments towards object detection widely studied are uncertain and dynamic mobility pattern, occlusion pattern, illumination issues, etc. [3]. Existing research work has also reported the usage of single and multi-camera in order to perform tracking and identification of an object. However, this is not at all an easier task to perform recognition of an object with higher accuracy. Hence, adoption of machine learning mechanism has become inevitable in this regards [4]. The contribution of machine learning approach is to offer more decision making for better identification, classification, and clustering operation in object detection [5]. However, there is also another field of advancement in object detection i.e. event detection which is a superset of object detection [6]. Majority of the implementation of object detection focuses more on foreground while event detection mechanism requires equal emphasis on both foreground and background to understand [7]. Still, the process of event detection is quite a challenging task because of following research question viz. 1) how to model an event discretely. We have observed that various researchers have modeled the event considering the supervised learning algorithm with higher emphasis on accuracy in identification process [8]. However, we find that accuracy is not found to be much better keeping it in balance with the computational demands. This process of event detection becomes further more challenging if the criticality of the event has to be determined. From application viewpoint, it is essential for the application to take decision based on the context of the scene, which is another challenging task [9]. It is because there are multiple forms of uncertainty associated in extracting contextual information from the event detection. Hence, the flow of the application design should be to prioritize on object detection followed by identification of possible event-based information and then to work on extracting amount of abnormality to confirm the context of the scene [10]. Hence, abnormality over the event-based information is one of the most important decision-making factors towards confirming false or true positive of the object detection.

Therefore, the proposed system discusses a novel modeling of a system that is capable of precisely identifying the abnormality of an event from a regular crowded scene. The significant novelty of the proposed system is to model an unsupervised learning technique and usage of a repository system that offers a superior mapping policy of the extracted regions for better identification performance.

The next Section II presents a discussion on related works followed by brief outlining of associated problems in it in Section III. Research methodology adopted to solve the problem is discussed in Section IV followed by an illustration of Algorithm implementation in Section V. Results obtained and discussion of the graphical outcome is carried out in Section VI and finally the conclusive remarks is given in Section VII.

\section{RELATED TEChNiQues}

This section discusses the existing approaches that deal with the investigation of the mobility behavior of an object on various scenarios. Existing techniques have witnessed usage of conventional object detection schemes that are quite high in number followed by research towards event detection method and abnormal event detection mechanism. 
The work carried out by Cao et al. [11] has used total variation method where a spatial continuity based approach is used for marking foreground. The outcome has exhibited a promising accuracy performance. Guan et al. [12] have used a dictionary-based mechanism (i.e., a bag of words) to perform detection of the objects in the road using semantics. However, the technique consumes maximum time for processing the outcome. Hu et al. [13] have used saliency features and low ranking representation to perform identification of moving object. Although the technique is highly comprehensive for moving object detection, it offers complexity. Kelantan et al. [14] have emphasized on identifying moving objects in multiple numbers using adjacency graphs. The system has used multi-graph matching policy as well as used labeling of the regions to perform detection of objects. Kang and Zhu [15] have adopted compressive sensing mechanism where circulate sampling mechanism is used for obtaining samples followed by a typical reconstruction method of the foreground. Usage of search optimization method is a witness in work carried out by Lee et al. [16]. The authors have constructed saliency map using a genetic algorithm to track the movement of an object. Tested on three different datasets, the outcome showed more than $94 \%$ of accuracy. Luo and Lai [17] have used localization factor as well as mapping attribute to perform identification of a moving object. The study contributes to the identification of an object from different image feed extracted from multiple sensors. Panda and Meher [18] have presented a technique for background subtraction using color difference histogram to control false errors. The authors have also used a fuzzy cmeans algorithm to minimize the dimensionality problems associated with histogram computation. Park et al. [19] have presented a k-nearest clustering mechanism on stereo images for identification of moving object. Adoption of a temporal factor is reported in the research carried out by Wang et al. [20] where the detection model is completely free from any dependencies on background modeling. The technique uses entropy and uses saliency map to perform identification of an object. Wu et al. [21] have used subtraction mechanism for background using singular value decomposition followed by adaptive thresholding. The technique uses fast in painting for reconstructing an image. Yeh et al. [22] have used hysteresis threshold technique to perform identification of mobile objects. Incorporation of the pyramidal feature is seen in the work carried out by Yuan et al. [23]. The presented paper has used the magnitude of difference and structural description of specific context. The work entirely emphasizes on the feature section process to find lesser processing time involved. Usage of Bayesian approach for a similar reason was seen in the work of Zhang et al. [24].

Literature has a good amount of work towards object detection system; however, there are also certain closer attempts towards event detection system. A study in such direction was carried out by Cosar et al. [25] where both problems of analysis of behavior as well as abnormal detection of behavior are jointly addressed using trajectory and pixelbased information. The technique also implements clustering mechanism on the analyzed grid to perform detection. Wang and Ji [26] have presented a machine learning approach along with semantics to perform event recognition. A priming contextual framework is designed for this purpose. Wen et al.
[27] have implemented the standard Gaussian model to obtain better tracking points for better response timing of event detection. A simple optimization process towards object detection is presented for better optical flow. Xian et al. [28] have used Fisher Vector to perform extraction of features (lowlevel) to perform event detection using the random forest. A standard test-bed is utilized to perform the assessment. There is very less number of studies that have focused on event detection in recent times.

Still, some of the researchers have tried their best to consider the further challenging problem of abnormality involved in event detection. Bae et al. [29] have presented a technique of identifying abnormal mobility of an object using partial trajectory-based information from the cluttered scene. However, the accuracy is not found to have better improvement irrespective of a better approach. Similar problems have been addressed by Chen et al. [30] using a nonconventional acceleration based framework. However, the technique cannot support the dynamic response of detection required for real-time performance. Fu et al. [31] have used feature learning system to identify the trajectories of an object. The presented technique is used for minimizing the space of search as well as a collaborative algorithm is presented to enhance the accuracy performance of abnormality detection process. Wang and Snoussi [32] have presented a unique classification technique using a conventional supervised learning algorithm to perform identification of the abnormal event. Tao et al. [33] have used semantics to perform identification of an abnormal event for a given scene using slow feature analysis. Zhang et al. [34] have considered compression and detection problem together on multimedia contents. Levy et al. [35] have developed a dataset that is meant for experimenting various identification of abnormal events in real-time. The work carried out by Yu et al. [36] has used sparsity-based approach along with gradient feature to offer better reconstruction method. The next section discusses problems being identified from existing literature.

\section{PROBLEM IDENTIFICATION}

The identified problems after reviewing the existing system on object and event detection are as follows:

- Lesser Improvement in Abnormal Movement Modeling: Majority of the existing study has no direct representation or standard definition of the abnormal event in object detection. The abnormality is a contextual term associated with the occurrence of the less probable appearance of object behavior for a given scene. Existing implementation ignores modernizing the techniques in the object detection scheme and directly works on event detection and hence the granularity in detection performance misses out.

- More Focus on Accuracy and Less on Complexity: Existing schemes mainly deals with implementing either iterative or complex technique of optimization resulting in higher accuracy at the cost of complexity. The biggest complexity associated in this regard is to extract the information about the exact location which has error-free information about the abnormal events. This result is good accuracy performance and lower 
complexity mitigation performance at the same time. This also results in a lower response time of the detection, which makes it less suitable for online schemes.

- Less Utilization of Spatio-Temporal Factor: Usage of time and space-related factors are very useful to understand the optical flow of an object. However, the mathematical modeling has the lesser inclusion of these factors jointly those results in ignorance towards the timely response of the identification modeling. This operation of emphasizing any one of the factors results in better accuracy but lowers down response time.

- Dominancy of Supervised Algorithm: Usage of supervised algorithms offers good convergence performance and better flexibility of achieving research goals; however, they also offer uncertainty to be applied in a real-time environment. On the other hand, adoption of unsupervised learning algorithms is quite challenging to build but once built than they can also be used for online or real-time environmental usage. However, it is a computationally challenging task to develop an unsupervised learning algorithm for dynamic object detection and event modeling.

It is essential to understand that modeling of abnormal event behavior is a combined study of behavioral analysis along with event modeling. The significant research problem is to jointly model abnormality event detection along with behavioral analysis. Because there has been enough work already carried out in behavioral analysis on the crowded scene, the focus will be narrowed down to abnormality event detection. Therefore, the research problem can be stated as "To design a framework for facilitating unsupervised modeling of abnormal event detection on a crowded scene."

\section{Proposed Methodology}

There are multiple impediments towards identifying an object in a precise manner. Our prior implementation has offered various techniques to deal with identification, recognition, tracking related issues for various dynamic scenarios of an object [37]-[40]. This part of the proposed system contributes to deal with abnormal event detection with the dynamic mobility of an object within it. The architecture of the proposed system is highlighted in Fig. 1.

\begin{tabular}{|c||c|c|}
\hline \multicolumn{3}{|c|}{ Given Scene } \\
\hline \hline \multicolumn{2}{|c|}{ Spatial Location } \\
\hline \hline Algorithm for Region Extraction & Algorithm for Training \\
\hline \hline \begin{tabular}{c}
\hline $\begin{array}{c}\text { Region } \\
\text { Development }\end{array}$ \\
\hline $\begin{array}{c}\text { Repository } \\
\text { Construction }\end{array}$
\end{tabular} \\
\hline
\end{tabular}

Fig. 1. Architecture of proposed abnormal event detection.

The proposed system takes the input as a given scene from where the abnormal event has to be identified. It, therefore, emphasized on the region around the object. A region of the proposed system is defined as all the possible section within the frame which has been captured with the abnormal event. The proposed system extracts the spatial and temporal attributes which are further subjected to two explicit algorithms for region extraction and training respectively. An empiricalbased approach is utilized for developing such regions. The proposed implements a unique technique for classifying different forms of image-related attributes by considering them as string attributes called as a repository. It will mean that each specific attributes obtained dynamically obtained from the given scene are converted to strings that makes the process of classification much well structured. This process also assists in constructing region-wise repository that finally assists in extracting event attributes. However, the process of detection is finally subjected to unsupervised training process using radial basis function to further confirm the correctness of abnormal event identification. The complete training algorithm is constructed using probability theory where a statistical significance is used to determine if the identified event is significant or not. The applicability of the proposed system suits well in the crowded scene where certain behavior or pattern of object mobility is highly restricted. It could also be used for security monitoring in hospitals, dock yards, airport, mining fields to perform precise identification of any lifethreatening events. The complete implementation is carried out considering an analytical research methodology where the focus is laid to the region extraction and training. The next section outlines the algorithm implemented to perform abnormal event detection.

\section{ALGORITHM IMPLEMENTATION}

This section outlines the algorithm implemented for the purpose of identifying the abnormality event in the given scene of object detection. The proposed study considers a definitive region under which the complete evaluation is carried out by the given frame. The first algorithm is mainly responsible for extracting the region by considering the input to be size (size of region), $I_{\mathrm{r}, \mathrm{c}}$ (rsize of frame), $R$ (region), $\Omega$ (repository) is database, $I_{\mathrm{trn}}$ (trained image), $N$ (Number of Sequence), $\lambda$ (size of one region) that after processing leads to the outcome of $\Omega$ (repository of the region). These parameters are helpful to capture the particular image frame from the video frame. Using this particular region is observed for abnormal behavior detection. The steps of the algorithm are as follows:

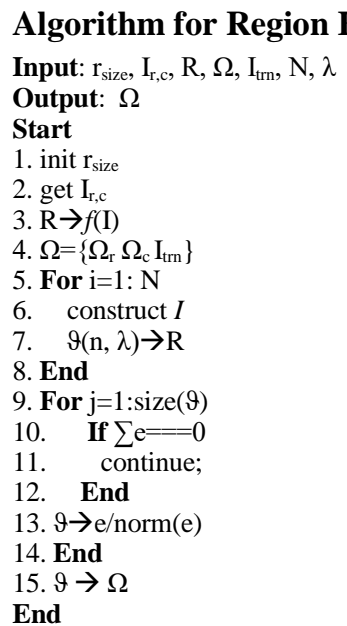


The above-mentioned algorithm takes the input of the sequence of images and proceeds to the construction of repository as well as region development. All the frames are read, and system initializes the image to be used for training $I_{\text {trn }}$ (Line-1, Line-2). The size of the frames is obtained and is extracted for some rows, column, and the index number of the cells. The algorithm than classifying the given frame using a function $f$ concerning equal region splits in orders to obtain core region $R$ (Line-3). The next part of the algorithm is to develop a repository $\Omega$ considering sub-repository $\Omega_{\mathrm{r}}$ and $\Omega_{\mathrm{c}}$, where $\Omega_{\mathrm{r}}$ and $\Omega_{\mathrm{c}}$ represent product of regions and size of region respectively (Line-4). The algorithm constructs region-

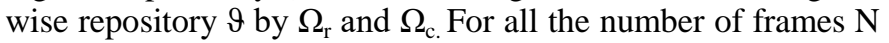
(Line-5), the proposed algorithm constructs region $\mathrm{R}$ by individual regions and size of one block $\lambda$. (Line-6). All the mean outcomes of the region-based repositories were obtained, i.e., $\sigma=$ mean $(\vartheta)$. For all the size of region-based repository $\vartheta$ (Line-9), an event attribute e is extracted from all the columnar elements of region-based repository $\vartheta$. In case there is no significant event (Line-10), the system continues to check for other regions (Line-11). The next event attribute $e$ is obtained as $e \rightarrow e-\sigma(\vartheta)$ followed by normalization of event attribute (Line-13). The updated information of the event attribute is stored back in a matrix that deposits $\vartheta$. The next part of the system is to apply the novel learning process to perform better object detection.

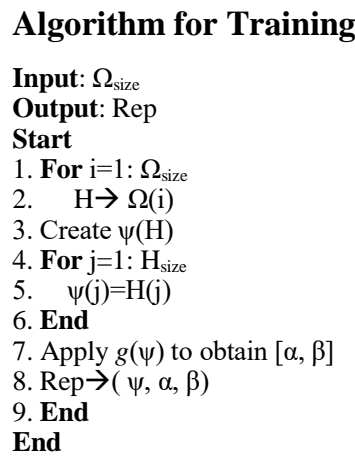

The outcome obtained from the prior algorithm of region extraction is used for as an input for the training process. For this purpose, a loop is constructed considering all the sizes of the repository $\Omega$ (Line-1). A temporary variable $H$ is constructed for obtaining all the size-related information from each repository $\Omega$ (Line-2). A structure $\psi$ is created that holds the information of sizes of matrix $\mathrm{H}$ (Line-3) and all the individual information retained in the matrix is transformed in a new matrix $\psi$ (Line-5). The next part of the algorithm applies an unsupervised learning algorithm in the form of a function $g(\psi)$ to obtain updated trained repositories of features, i.e., $\alpha, \beta$ (Line-7). The final construction of repository is carried out considering $\psi$ along with $\alpha, \beta$ (Line-8). The construction of the function $g$ is designed considering radial basis function as the kernel attribute. The next step is to perform detection of the abnormal event using test-frame sequence and followed by almost similar steps of selection of regions and training. However, the technique loads the repository outcomes and computes the probability of minimum distance between two data points and compared with the ground truth images. It applies the process of matrix decomposition to obtain the concatenated centroid position of the test mage followed by dilation operation on the ground truth image. Certain threshold is fixed to identify the possibilities of abnormalities on the given scene as the cumulative outcome of the proposed algorithm.

TABLE. I. LIST OF NOTATION

\begin{tabular}{|l|l|}
\hline$r_{\text {size }}$ & Size of the region \\
\hline$I_{r, c}$ & Size of the frame \\
\hline$R$ & Region \\
\hline$\Omega / \Omega_{\text {size }}$ & Repository, size of repository \\
\hline$I_{t r n}$ & Trained image \\
\hline$N$ & Number of sequences \\
\hline$A$ & Size of one region \\
\hline$R e p$ & Trained data \\
\hline$e$ & Event attribute \\
\hline
\end{tabular}

\section{RESULT ANALYSIS}

The proposed system is assessed considering the UCSD pedestrian dataset [41]. In this, MATLAB is used for image isolation from the video. The data set consists of more than 1000 image sequences with both test and trained images. The time frame for scene change is indicated for $15 \mathrm{sec} / \mathrm{frame}$. Each frame shows a lane where people are found to walking in opposite direction, and their behavior of crowd cannot be predicted to a particular pattern. At the same time, we consider an event to be taken place when any object, e.g., cart, bicycle, 4-wheeler, etc. is found to be moving within the visual scope of the lane.

The implementation of the proposed study is carried in Matlab and using the similar data; the study outcome is compared with similar existing techniques dealing with event detection concerning accuracy and computation time. The existing studies compared with are the work carried out by Saligrama et al. [42] - LSA, Roshtkhari et al. [43] - ODABD, Mahadevan et al. [44] - AD, Bertini et al. [45] - NPA, and Reddy et al. [46] - CBA.

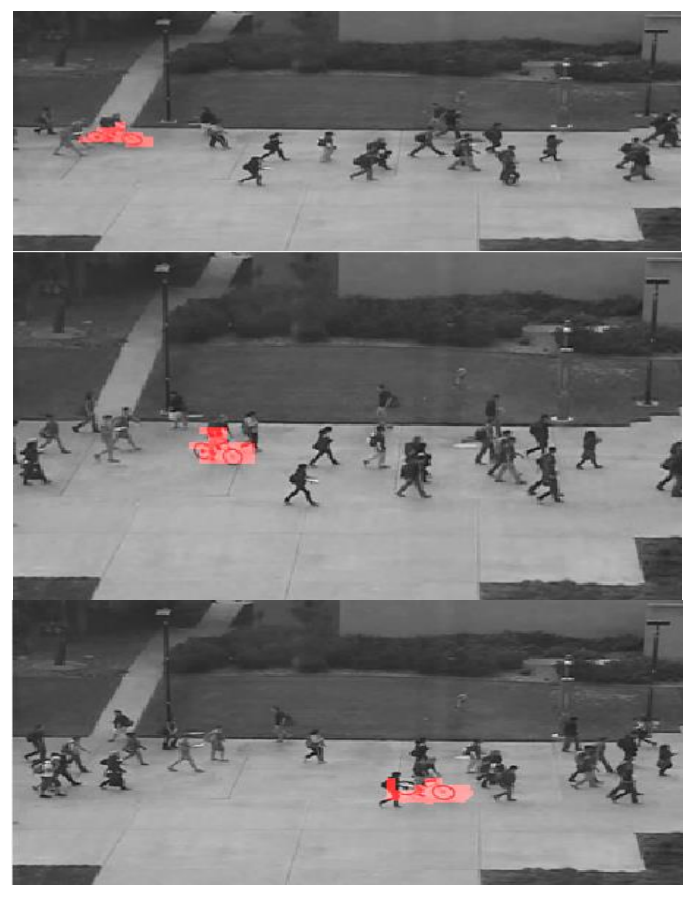

Fig. 2. Visual capturing of abnormal events. 


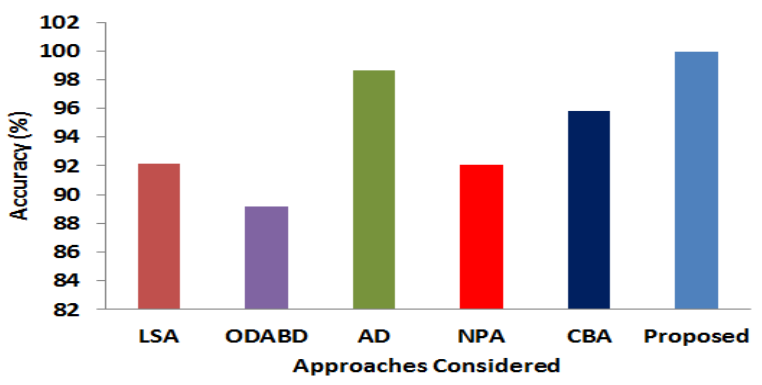

Fig. 3. Comparative analysis of accuracy.

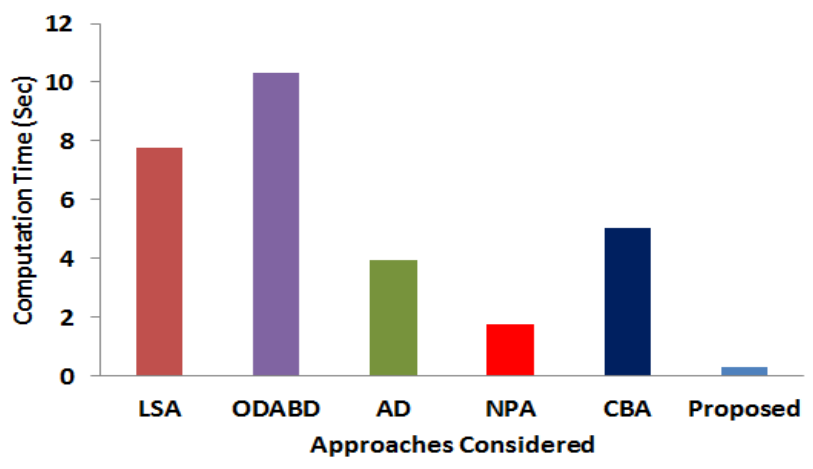

Fig. 4. Comparative analysis of computation time.

The study outcome shows that proposed system excels better event of abnormality detection performance as compared to all the existing system. The prime reason for enhanced accuracy of the proposed system is the granularity in the approach for event detection using the concept of matrix factorization. Another essential contribution is the novel learning algorithm that exploits the histogram attributes for extracting the information of any unusual object appearance for a given scene and can easily justify and segregate the usual to unusual appearance of the object. This will also mean that proposed system uses smaller time frame for computing the sophisticated behavior of the given crowded scene also. The final reason for this outcome is discrete modeling of time, and spatial attributes for better context-based information can be extracted for incorporating granularity in abnormal event detection system. At the same time, the algorithm offers faster response time in comparison to the existing system.

\section{CONCLUSION}

With the proliferation of video surveillance system, it is quite imperative that there have been some significant proposals for the object as well as event detection scheme. However, after reviewing the existing system, we find that there are some significant problems, e.g., 1) Lesser Improvement in Abnormal Movement Modeling; 2) More Focus on Accuracy and Less on Complexity; 3) Less Utilization of Spatio-Temporal Factor; and 4) Dominancy of Supervised Algorithm. This problem leads to less emphasize on the modeling aspect of the abnormality factor, which is always regarding probability. The proposed system considers that if any alienated object is found to display a mobility behavior which is completely different from what has been captured till the time instance is called as an abnormality. A good utilization of this scheme could be to monitor the patient in coma stage. A sudden movement captured from such patient is treated as an abnormal event that prompts for alarm. Similarly, it could be used in industrial monitoring and other traffic-related monitoring system too. The presented system applies a mechanism where local patterns are emphasized on each region for a given frame using histograms. The proposed system also applies probability theory for modeling using temporal and spatial factor using unsupervised learning algorithm.

The proposed method can be used to the method can be used for surveillance system of a location, mall, military applications and this research can be used as benchmarks for further improvement in security systems.

\section{REFERENCES}

[1] Bir Bhanu, Chinya V. Ravishankar, Amit K. Roy-Chowdhury, Hamid Aghajan, Demetri Terzopoulos, Distributed Video Sensor Networks, Springer Science \& Business Media, 2011

[2] C. R. del-Blanco, F. Jaureguizar and N. Garcia, "An efficient multiple object detection and tracking framework for automatic counting and video surveillance applications," in IEEE Transactions on Consumer Electronics, vol. 58, no. 3, pp. 857-862, August 2012.

[3] S. Qiu, G. Wen, and Y. Fan, "Occluded Object Detection in HighResolution Remote Sensing Images Using Partial Configuration Object Model," in IEEE Journal of Selected Topics in Applied Earth Observations and Remote Sensing, vol. 10, no. 5, pp. 1909-1925, May 2017

[4] H. Song, Z. Liu, H. Du, G. Sun, O. Le Meur and T. Ren, "Depth-Aware Salient Object Detection and Segmentation via Multiscale Discriminative Saliency Fusion and Bootstrap Learning," in IEEE Transactions on Image Processing, vol. 26, no. 9, pp. 4204-4216, Sept. 2017.

[5] A. T. Angonese and P. F. Ferreira Rosa, "Multiple people detection and identification system integrated with a dynamic simultaneous localization and mapping system for an autonomous mobile robotic platform," 2017 International Conference on Military Technologies (ICMT), Brno, 2017, pp. 779-786.

[6] A. Bauer and Y. Fischer, "Object-event graph matching for complex activity recognition," 2011 IEEE International Multi-Disciplinary Conference on Cognitive Methods in Situation Awareness and Decision Support (CogSIMA), Miami Beach, FL, 2011, pp. 88-93.

[7] H. Shin, J. Gwak, J. Yu and M. Jeon, "Feature flow-based abnormal event detection using a scene-adaptive cuboid determination method," 2016 International Conference on Control, Automation and Information Sciences (ICCAIS), Ansan, 2016, pp. 205-209.

[8] Y. Zhou, R. Arghandeh, I. Konstantakopoulos, S. Abdullah and C. J. Spanos, "Data-driven event detection with partial knowledge: A Hidden Structure Semi-Supervised learning method," 2016 American Control Conference (ACC), Boston, MA, 2016, pp. 5962-5968.

[9] B. Andò, S. Baglio, C. O. Lombardo and V. Marletta, "An Event Polarized Paradigm for ADL Detection in AAL Context," in IEEE Transactions on Instrumentation and Measurement, vol. 64, no. 7, pp. 1814-1825, July 2015

[10] T. H. Huang, V. Nikulin, and L. B. Chen, "Detection of Abnormalities in Driving Style Based on Moving Object Trajectories without Labels," 2016 5th III International Congress on Advanced Applied Informatics (III-AAI), Kumamoto, 2016, pp. 675-680.

[11] X. Cao, L. Yang and X. Guo, "Total Variation Regularized RSPCA for Irregularly Moving Object Detection Under Dynamic Background,” in IEEE Transactions on Cybernetics, vol. 46, no. 4, pp. 1014-1027, April 2016.

[12] H. Guan, Y. Yu, J. Li and P. Liu, "Pole-Like Road Object Detection in Mobile LiDAR Data via Supervoxel and Bag-of-Contextual-VisualWords Representation," in IEEE Geoscience and Remote Sensing Letters, vol. 13, no. 4, pp. 520-524, April 2016.

[13] W. Hu, Y. Yang, W. Zhang, and Y. Xie, "Moving Object Detection Using Tensor-Based Low-Rank and Saliently Fused-Sparse 
Decomposition," in IEEE Transactions on Image Processing, vol. 26, no. 2, pp. 724-737, Feb. 2017

[14] B. Kalantar, S. B. Mansor, A. Abdul Halim, H. Z. M. Shafri and M. Zand, "Multiple Moving Object Detection From UAV Videos Using Trajectories of Matched Regional Adjacency Graphs," in IEEE Transactions on Geoscience and Remote Sensing, vol. 55, no. 9, pp. 5198-5213, Sept. 2017.

[15] B. Kang and W. P. Zhu, "Robust moving object detection using compressed sensing," in IET Image Processing, vol. 9, no. 9, pp. 811819, 92015.

[16] G. Lee, R. Mallipeddi, G. J. Jang and M. Lee, "A Genetic AlgorithmBased Moving Object Detection for Real-time Traffic Surveillance," in IEEE Signal Processing Letters, vol. 22, no. 10, pp. 1619-1622, Oct. 2015

[17] R. C. Luo and C. C. Lai, "Multisensor Fusion-Based Concurrent Environment Mapping and Moving Object Detection for Intelligent Service Robotics," in IEEE Transactions on Industrial Electronics, vol. 61, no. 8, pp. 4043-4051, Aug. 2014.

[18] D. K. Panda and S. Meher, "Detection of Moving Objects Using Fuzzy Color Difference Histogram-Based Background Subtraction," in IEEE Signal Processing Letters, vol. 23, no. 1, pp. 45-49, Jan. 2016.

[19] J. Park, J. H. Yoon, M. G. Park and K. J. Yoon, "Dynamic Point Clustering with Line Constraints for Moving Object Detection in DAS," in IEEE Signal Processing Letters, vol. 21, no. 10, pp. 1255-1259, Oct. 2014.

[20] Z. Wang, K. Liao, J. Xiong and Q. Zhang, "Moving Object Detection Based on Temporal Information," in IEEE Signal Processing Letters, vol. 21, no. 11, pp. 1403-1407, Nov. 2014.

[21] Y. Wu, X. He and T. Q. Nguyen, "Moving Object Detection With a Freely Moving Camera via Background Motion Subtraction," in IEEE Transactions on Circuits and Systems for Video Technology, vol. 27, no. 2, pp. 236-248, Feb. 2017.

[22] C. H. Yeh, C. Y. Lin, K. Muchtar, H. E. Lai and M. T. Sun, "ThreePronged Compensation and Hysteresis Thresholding for Moving Object Detection in Real-Time Video Surveillance," in IEEE Transactions on Industrial Electronics, vol. 64, no. 6, pp. 4945-4955, June 2017.

[23] X. Yuan, X. Cao, X. Hao, H. Chen and X. Wei, "Vehicle Detection by a Context-Aware Multichannel Feature Pyramid," in IEEE Transactions on Systems, Man, and Cybernetics: Systems, vol. 47, no. 7, pp. 13481357, July 2017.

[24] X. Zhang, C. Zhu, S. Wang, Y. Liu and M. Ye, "A Bayesian Approach to Camouflaged Moving Object Detection," in IEEE Transactions on Circuits and Systems for Video Technology, vol. 27, no. 9, pp. 20012013, Sept. 2017.

[25] S. Coşar, G. Donatiello, V. Bogorny, C. Garate, L. O. Alvares and F. Brémond, "Toward Abnormal Trajectory and Event Detection in Video Surveillance," in IEEE Transactions on Circuits and Systems for Video Technology, vol. 27, no. 3, pp. 683-695, March 2017.

[26] X. Wang and Q. Ji, "Hierarchical Context Modeling for Video Event Recognition," in IEEE Transactions on Pattern Analysis and Machine Intelligence, vol. 39, no. 9, pp. 1770-1782, Sept. 1, 2017.

[27] J. Wen, Z. Lai, Z. Ming, W. K. Wong, and Z. Zhong, "Directional Gaussian Model for Automatic Speeding Event Detection," in IEEE Transactions on Information Forensics and Security, vol. 12, no. 10, pp. 2292-2307, Oct. 2017.

[28] Y. Xian, X. Rong, X. Yang and Y. Tian, "Evaluation of Low-Level Features for Real-World Surveillance Event Detection," in IEEE Transactions on Circuits and Systems for Video Technology, vol. 27, no. 3, pp. 624-634, March 2017.
[29] G. T. Bae, S. Y. Kwak and H. R. Byun, "Motion pattern analysis using partial trajectories for abnormal movement detection in crowded scenes," in Electronics Letters, vol. 49, no. 3, pp. 186-187, Jan. 312013.

[30] C. Chen, Y. Shao and X. Bi, "Detection of Anomalous Crowd Behavior Based on the Acceleration Feature," in IEEE Sensors Journal, vol. 15, no. 12, pp. 7252-7261, Dec. 2015.

[31] P. Fu, H. Wang, K. Liu, X. Hu and H. Zhang, "Finding Abnormal Vessel Trajectories Using Feature Learning," in IEEE Access, vol. 5, no. , pp. 7898-7909, 2017.

[32] T. Wang and H. Snoussi, "Detection of Abnormal Visual Events via Global Optical Flow Orientation Histogram," in IEEE Transactions on Information Forensics and Security, vol. 9, no. 6, pp. 988-998, June 2014.

[33] Ye Tao, Ye Jin and Peng Liu, "A high semantic representation for abnormal events detection in crowded scenes," 2016 7th IEEE International Conference on Software Engineering and Service Science (ICSESS), Beijing, 2016, pp. 17-20.

[34] Y. Zhang and H. Chao, "Abnormal Event Detection in Surveillance Video: A Compressed Domain Approach for HEVC," 2017 Data Compression Conference (DCC), Snowbird, UT, 2017, pp. 475-475.

[35] R. Leyva, V. Sanchez and C. T. Li, "The LV dataset: A realistic surveillance video dataset for abnormal event detection," 20175 th International Workshop on Biometrics and Forensics (IWBF), Coventry, 2017,

[36] B. Yu, Y. Liu and Q. Sun, "A Content-Adaptively Sparse Reconstruction Method for Abnormal Events Detection With Low-Rank Property," in IEEE Transactions on Systems, Man, and Cybernetics: Systems, vol. 47, no. 4, pp. 704-716, April 2017.

[37] Pushpa, D., H.S. Sheshadri, "Homography based Multiple Moving Objects Using Multi-Dimensional Event Feeds," International Journal of Computer Science and Information Technology Security, Vol. 3, No.5, October 2013

[38] Pushpa D. and H. S. Sheshadri, "Scale-invariant object identification for jointly addressing issues in moving object and background," 2015 International Conference on Emerging Research in Electronics, Computer Science and Technology (ICERECT), Mandya, 2015, pp. 168173.

[39] Pushpa D. and H S Sheshadri. Article: Multiple Object Detection and Tracking in Cluttered Region with Rational Mobile Area. International Journal of Computer Applications 39(10):14-17, February 2012

[40] Pushpa D. and H S Sheshadri, "Semantic Analysis of Precise Detection Rate in Multi-Object Mobility on Natural Scene Using Kalman Filter," Springer, Emerging Research in Electronics, Computer Science and Technology, pp 217-226, 2013

[41] "UCSD Anomaly Detection Dataset," http://www.svcl.ucsd.edu/projects/anomaly/dataset.htm, Retrieved $24^{\text {th }}$ October 2017

[42] V. Saligrama and C. Zhu, "Video anomaly detection based on local statistical aggregates," in Proc. Computer Vision and Pattern Recognition, 2012.

[43] M. J. Roshtkhari and M. D. Levine, "Online dominant and anomalous behavior detection in videos," in Proc. Computer Vision and Pattern Recognition, 2013

[44] V. Mahadevan, W. Li, V. Bhalodia, and N. Vasconcelos, "Anomaly detection in crowded scenes," in Proc. Computer Vision and Pattern Recognition, 2010

[45] M. Bertini, A. Del Bimbo, and L. Seidenari, "Multi-scale and realtime non-parametric approach for anomaly detection and localization," in CVIU, 2012.

[46] V. Reddy, C. Sanderson, and B. C. Lovell, "Improved anomaly detection in crowded scenes via cell-based analysis of foreground speed," size and texture," in Proc. Computer Vision and Pattern Recognition Workshops, 2011 